\title{
Editorial "Discrepancy Between the Clinical and Final Pathological Findings of Lymph Node Metastasis in Superficial Esophageal Cancer"
}

\author{
Rian M. Hasson, MD, and Joseph D. Phillips, MD \\ Section of Thoracic Surgery, Department of Surgery, Dartmouth-Hitchcock Medical Center, Lebanon, NH
}

While the presence of lymph node metastasis in esophageal cancer continues to be one of the most important predictors of long-term prognosis, accurate identification by clinical means remains challenging. ${ }^{1,2}$ This is especially true for patients with T1a and T1b tumors for which correct assessment of lymph node involvement is paramount to prescription of the appropriate treatment. In the manuscript entitled, "Discrepancy between the clinical and final pathologic findings of lymph node metastasis in superficial esophageal cancer," Aoyama et al. evaluate the inconsistency between the clinical and pathologic diagnoses of lymph node invasion in patients with early-stage esophageal cancer. ${ }^{3}$ In addition, they describe the characteristics of lymph node metastasis that limit clinical detection with current screening modalities and conclude by recommending surgeons express caution before reducing the extent of lymph node dissection for early esophageal cancers in patients with clinically node-negative disease. This represents a thoughtful and well-researched recommendation for surgeons performing surgical resection. However, the findings of this study raise additional questions regarding patients with early stage tumors that are treated endoscopically and do not undergo lymph node resection. Perhaps, have we been overestimating our ability to properly stage these cancers?

Today, increased utilization of esophageal sparing procedures that do not include lymph node dissection are not only preferred but are standard-of-care for early-stage

(C) Society of Surgical Oncology 2019

First Received: 29 May 2019;

Published Online: 21 June 2019

R. M. Hasson, MD

e-mail: Rian.M.Hasson@Hitchcock.Org esophageal cancers. ${ }^{4}$ However, dependence on clinical measures alone to assess lymph node status makes appropriate patient selection challenging. ${ }^{5}$ The reasons are multifactorial, but are centered on the lack of reliable preexcision molecular, immunohistochemical, and biologic predictive markers of lymph node metastasis in T1 esophageal cancer. ${ }^{5}$ More importantly, clinically based lymph node assessment modalities have a low diagnostic performance for N1 disease. Taking this into consideration and given Aoyama et al.'s findings, do we need to revisit current diagnostic and lymph node staging methods for nonsurgical candidates?

To better understand the current shortcomings, it is imperative to understand the potential risk of occult lymph node metastasis and the resulting change in prognosis. "Subclinical metastasis," "micrometastasis," or "tumor cell microinvolvement" are all terms that have been used to describe disseminated cancer cells detected by immunochemical means in lymph nodes. ${ }^{6}$ Clarification of this term was attempted by the Union for International Cancer Control (UICC) by making a distinction between "isolated tumor cells" and "micrometastasis." Micrometastasis are defined as being " $<2 \mathrm{~mm}$ in greatest dimension; in contact with a vessel wall, proliferating, and usually associated with a stromal reaction". ${ }^{6}$ In contrast, isolated tumor cells are defined as "clusters $<0.2 \mathrm{~mm}$ or single tumor cells without any of the above characteristics, whose presence can be determined by immunochemistry, immunocytochemistry or molecular methods such as flow cytometry or polymerase chain reaction". ${ }^{6}$ To date, data suggest that it is possible to detect disseminated tumor cells in lymph nodes previously considered free of metastases by using conventional histopathologic methods in many types of cancer. ${ }^{6}$ However, attempts to define their presence in esophageal cancer by clinical means and determine significance have demonstrated less satisfying results and 
outcomes, respectively. McGuill et al. set out to quantify the impact of occult lymph node metastasis on disease-free survival in cases of early esophageal cancer staged by pathologic means as lymph node metastasis-free $(\mathrm{pN} 0){ }^{6}$ Their meta-analysis suggested that occult lymph node metastasis has a "significant detrimental impact" on prognosis in node-negative cancers of the esophagus or gastro-esophagus. Furthermore, their results were not meaningfully affected by the mean number of lymph nodes examined, variations in the number of sections examined per node or block, exclusion of trials of poorer quality, or choice in statistical method. ${ }^{6}$ More recently, Lütken et al. reviewed the impact of isolated tumor cells in regional lymph nodes in patients with adeno- and squamous cell carcinoma of the esophagus and esophagogastric junction. ${ }^{7}$ Specifically, they reviewed 5 studies, including 434 pNOpatients of which 88 patients had isolated tumor cells (ITC) on more extensive review. This rate of ITC varied from $8 \%$ to $56 \%$ between studies, and the majority of studies found a significant prognostic impact of ITC with the largest difference in 5-year survival being $33 \%$ for patients with ITC compared with $60 \%$ for those without ITC. ${ }^{7}$ Fortunately, in Aoyama's study, the "missed" areas of disease were all classified as micrometastasis or larger.

Because the consequences of missed lymph node involvement are significant, are there risk factors for occult metastatic disease that can be used to more accurately clinically stage patients? Dubecz et al. evaluated 1225 patients with $\mathrm{T} 1 \mathrm{a}$ and $\mathrm{T} 1 \mathrm{~b}$ lesions to determine the prevalence and predictors of lymph node metastases in patients with pT1 carcinoma of the esophagus and gastric cardia. ${ }^{8}$ They found that tumor size greater than $1 \mathrm{~cm}$, infiltration of submucosa, and poor tumor differentiation were independently associated with the risk of nodal disease, and the presence of lymph node metastasis without these three risk factors was only $4.8 \%{ }^{8}$ Furthermore, positive lymph node status was associated with worse overall 5-year survival in esophageal adenocarcinoma (EAC) (N0 vs. $\mathrm{N}+: 78 \%$ vs. $52 \%$ ) and adenocarcinoma of the esophagogastric junction (AEG) (N0 vs. $\mathrm{N}+: 83 \%$ vs. $44 \%$ ) but did not have a significant effect on the long-term survival of patients with squamous cell carcinoma (SCC). ${ }^{8}$

Endoscopic mucosal resection (EMR) and endoscopic submucosal dissection (ESD) embody a long sought after, minimally invasive treatment of early cancer. ${ }^{9,10}$ Offering the benefits of organ preservation without the long-term consequences of radical resection, it can be performed with low morbidity. ${ }^{5}$ Current National Comprehensive Cancer Network (NCCN) guidelines recommend EMR for T1s, T1a, and highly selected T1b esophageal cancers. ${ }^{11}$ However, indiscriminant use of these techniques may lead to inferior long-term outcomes of patients with understaged esophageal cancer when unknown lymph node metastases are present and left behind. ${ }^{8,12,13}$

To address these concerns, in addition to the use of computed tomography (CT), positron emission tomography (PET)-CT, and endoscopic ultrasound with fine-needle aspiration (EUS-FNA), to stage early esophageal cancer, many have offered considerations for patients who are endoscopic resection candidates. ${ }^{14}$ First, one must pay close attention to low- and high-risk features of T1a and T1b tumors. For example, in T1a tumors with poor differentiation or size $\geq 2 \mathrm{~cm}$, esophagectomy should be considered. ${ }^{12}$ Conversely, in T1b tumors with low-risk features, including well differentiation, size $<2 \mathrm{~cm}$, and absent lymphovascular invasion, endoscopic resection should be considered and may be curative. ${ }^{12}$ Second, the combining of risk factors into a model-based scoring system to calculate the probability of the risk of lymph node metastasis in T1 esophageal adenocarcinoma has been proposed. ${ }^{14} \mathrm{Wu}$ et al. recently expounded this work by presenting a model demonstrating great promise in facilitating individualized risk for lymph node metastasis in patients with $\mathrm{T} 1$ squamous cell carcinoma to establish candidacy for endoscopic resection. ${ }^{16}$ Others appear to be following suit. ${ }^{15}$

Suggestions that are more controversial have included the use of adjuvant concurrent chemoradiotherapy in patients determined to have a high risk of lymph node metastasis who undergo endoscopic submucosal dissection. ${ }^{16}$ Additionally, the use of EMR as a diagnostic tool to better evaluate histologic features that might categorize the patient as high risk also has been proposed. ${ }^{17}$ Interestingly, there has been little study regarding the utility of isolated lymph node sampling/dissection in patients thought to have high-risk features who undergo endoscopic resection. Nevertheless, further study is needed before any of these more controversial ideas can be implemented in everyday practice.

Ultimately, when making treatment plans for early-stage esophageal cancer, clinicians must weigh the potential risks of perioperative morbidity and mortality against the possibility of understaging with a lack of pathologic lymph node evaluation. ${ }^{12}$ Keeping this in mind, Aoyama et al.'s findings are important for multiple reasons. First, they provide a key reminder that although significant advances have occurred with technology when diagnosing and staging esophageal cancer, our current modalities have limitations in clinically early-stage patients. Second, barring patient comorbidities and physical limitations, esophagectomy can always be utilized if pathology demonstrates high-risk features or there is uncertainty regarding the extent of the tumor or nodal involvement. Third, if esophagectomy is required, adequate lymph node 
procurement is the most accurate way to stage esophageal cancer and estimate prognosis in the postoperative setting. Fortunately, most endoscopic procedures are performed for cases of high-grade dysplasia and superficial cancer, and outcomes in patients who are properly staged are good. In the end, clinicians must use all of the clinical and diagnostic tools available (either in or out of the operating room) to accurately stage esophageal cancer to offer the best treatment that will limit recurrence, provide the lowest morbidity and mortality, and maximize quality of life.

\section{REFERENCES}

1. The Society of Thoracic Surgeons General Thoracic Surgery Database Task Force. The Society of Thoracic Surgeons composite score for evaluating esophagectomy for esophageal cancer. Ann Thorac Surg. 2017;103(5):1661-7.

2. Barbour, AP, Jones M, Brown I, Gotley DC, Martin I, Thomas J, Clouston A, Smithers BM. Risk stratification for early esophageal adenocarcinoma: analysis of lymphatic spread and prognostic factors. Ann Surg Oncol. 2010;17(9):2494-502.

3. Aoyama J, Kawakubo H, Mayanagi S, Fukada K, Nakamura R, Wada N, Suzuki T, Kameyama K, Kitagawa Y. Discrepancy between the clinical and final pathological findings of lymph node metastasis in superficial esophageal cancer. Ann Surg Oncol. 2019. (in press).

4. Sqourakis G, Gockel I, Lang H. Endoscopic and surgical resection of T1a/T1b esophageal neoplasms: a systemic review. World J Gastroenterol. 2013;19(9):1424-37.

5. Nobel T, Barbetta A, Hsu M, Tan KS, Sihag S, Bains MS, Jones $\mathrm{D}$, Molena D. Ongoing challenges in clinical assessment of nodal status in T1 esophageal adenocarcinoma. J Am Coll Surg. 2019. h ttps://doi.org/10.1016/j.jamcollsurg.2019.04.032.

6. McGuill MJ, Byrne P, Reynolds RJ. The prognostic impact of occult lymph node metastasis in cancer of the esophagus or esaphago-gastric junction: systemic review and meta-analysis. Dis Esophagus. 2008;21(3):236-40.

7. Lütken, CD, Fiehn AK, Federspiel B, Achiam MP. Impact of isolated tumor cells in regional lymph nodes in adeno- and squamous cell carcinoma of the esophagus and the esophagogastric junction-a systematic review. Pathol Res Pract. 2019;215(5):849-54.
8. Dubecz A, Kern M, Solymosi N, Schweigert M, Stein HJ. Predictors of lymph node metastasis in surgically resected T1 esophageal cancer. Ann Thoracic Surg. 2015;99(6):1879-86.

9. Ell C. Vakil N, Bergman J, et al. Curative endoscopic resection of early esophageal adenocarcinomas (Barrett's cancer). Gastrointest Endosc. 2007;65(1):3-10.

10. Ngamruengphong S, Wolfsen HC, Wallace MB. Survival of patients with superficial esophageal adenocarcinoma after endoscopic treatment vs. surgery. Clin Gastroenterol Hepatol. 2013;11(11):1423-9.

11. National Comprehensive Cancer Network. NCCN clinical practice guidelines in oncology: esophageal and esophagogastric junction cancers (Version 1.2017). https://www.nccn.org/profess ionals/physciain_gls/PDS/esophageal.pdf. Accessed 23 May 2019.

12. Newton AD, Predina JD, Xia L, Roses RE, Karakousis GC, Depmsey DT, Williams NN, Kucharczuk JC, Singhal S. Surgical management of early-stage esophageal adenocarcinoma based on lymph node metastasis risk. Ann Surg Oncol. 2018;25(1):318-25.

13. Rice TW, Rusch VW, Apperson-Hansen C, Allen MD, Chen LQ, Hunter JG, Kesler KA, Law S, Lerut TE, Reed CE, Salo JA, Scott WJ, Swisher SG, Watson TJ, Blackstone EH. Worldwide esophageal cancer collaboration. Dis Esophogus. 2009;22(1):1-8.

14. Lee L, Ronellenfitsch U, Hofstetter WL, Darling G, Gaiser T, Lipper C, Gilbert S, Seely AJ, Mulder DS, Ferri LE. Predicting lymph node metastasis in early esophageal adenocarcinoma using a simple scoring system. J Am Coll Surg. 2013;217(2):191-9.

15. Zhou Y, Du J, Wang Y, Li H, Ping G, Luo J, Chen L, Zhang S, Wang W. Prediction of lymph node metastatic status in superficial esophageal squamous cell carcinoma using an assessment model combining clinical characteristics and pathologic results: a retrospective cohort study. Int J Surg. 2019;66:53-61.

16. Kim HK, Ko, WJ, Kwon C-II, Song GW, Yoo IK, Song JH, Kim HS, Cho JY. Endoscopic submucosal dissection followed by concurrent chemoradiotherapy in patients with early esophageal cancer with a high risk of lymph node metastasis. Clin Endosc. 2019. https://doi.org/10.5946/ce.2018.176.

17. Pouw RE, Heldoorn N, Herrero A, et al. Do we still need EUS in the work-up of patients with early esophageal neoplasia? A retrospective analysis of 131 cases. Gastrointest Endosc. 2011;73(4):662-8.

Publisher's Note Springer Nature remains neutral with regard to jurisdictional claims in published maps and institutional affiliations. 